\title{
Crowdworking: working with or against the crowd?
}

\author{
Georg Jäger ${ }^{1}$ (D) $\cdot$ Laura S. Zilian ${ }^{1} \cdot$ Christian Hofer $^{1} \cdot$ Manfred Füllsack $^{1}$
}

Received: 11 February 2019 / Accepted: 5 September 2019 / Published online: 24 September 2019 (c) The Author(s) 2019

\begin{abstract}
Our current labour market is affected by massive changes like digitalization, automation and globalization, which gives rise to completely new forms of generating income. One such innovative idea is crowdworking, where many people (a so-called crowd) work on individual tasks for a firm in a way similar to a self-employed freelancer. This form of occupation is a recent development but gains acceptance, esteem and relevance quite rapidly. The risk potential for wage dumping and (self-) exploitation is still unknown. A crucial, but often neglected fact about crowdworking is that it exists in many variants which have completely different properties. We investigate how much these distinct versions of crowdworking differ by using an agent-based computer simulation. Wages, job security, workforce composition and other relevant indicators are calculated by simulating the micro scale to gain aggregated information on the macro-scale. We find that there is a significant difference between the versions of crowdworking. Our main finding is that especially variants where the crowdworkers are able to set their own wages are susceptible to wage dumping. Simulations suggest that this phenomenon is independent of the specifics of the labour market but rather a fundamental property of those variants of crowdworking.
\end{abstract}

Keywords Crowdworking · Gig economy · Agent-based model · Wage dumping • Digitalization · Labour market · Working poor · Exploitation

JEL Classification C63 $\cdot$ J24 $\cdot$ J28

\section{Introduction}

Digitalization, automation and globalization rapidly change the current labour market. These changes not only influence wages, unemployment and working conditions, but also our general understanding of what labour is and how income can and should

Georg Jäger

georg.jaeger@uni-graz.at

1 University of Graz, Merangasse 18, 8010 Graz, Austria 
be obtained. Society is struggling with these changes. We can observe many grave problems in that context that can be attributed to a dated system of how to distribute jobs, income and resources.

It is therefore paramount to investigate how society might react to these significant changes. One of the ways to adapt to the situation of the job market, that is not only currently popular, but also gains importance quite rapidly is known as crowdworking. However, the long-term effects and potential risks of crowdworking are not very well understood. In order to fill this research gap, we will use an agent-based computer simulation and compare various forms of crowdworking.

\subsection{The transformation of the labour market}

One main reason for the change of our current labour market is automation and digitalization. Traditionally, automation replaced manual, routine labour, i.e. processes that require not much creativity. Today, with the advance of digitalization and artificial intelligence, many other jobs are susceptible to replacement and this process is likely to continue in the future. Much research is dedicated to the amount of automation we have to expect, and it is predicted that up to $50 \%$ of the jobs, currently performed by humans, will be performed by robots or digital machines in the next 20 years (Frey and Osborne 2017; Brynjolfsson and McAfee 2014; Ford 2015; Düll et al. 2016).

Another big factor is globalization and the related topic of labour migration (VoglerLudwig et al. 2015; ILO 2016). Many corporations that operate internationally choose to offer their jobs in regions where the wages are cheaper or which have other financial advantages for them. With increasing globalization, this is done more often and our traditional labour market has difficulties to cope with this situation.

These difficulties become apparent by investigating relevant indicators like unemployment. Even within the European Union the harmonized unemployment rate is around $8 \%$, and there are no signs that this is just a temporary situation (OECD 2017). We can also see that regular employment with a secure income is not the norm any longer. Currently, there are many areas where precarious work forms are common (Deranty 2008; Bescherer 2009; Scherschel et al. 2012). The resulting income insecurities affect a significant fraction of the population.

However, not all changes and tendencies are negative. People are increasingly interested in leisure time (Boeri et al. 2008). They are beginning to choose their job not only based on financial aspects, but also on other factors that might increase their happiness. This world view is steadily gaining importance. There are also efforts to establish a better work-life-balance, in the sense that work should only be a part of who we are, but financial gain not the ultimate goal to which we dedicate our lives (Rosa 2005, 2010; Reheis 2009).

This phenomenon that there are huge difficulties for many people to obtain work, while others have the impression that they work too much is often called dualization (Emmenegger 2012). At first, it seems paradoxical, but it is only a sign that there are significant problems with our contemporary labour market, where unemployed, precariously employed and even securely employed people feel the need to change something about their current situation. This desire for change leads to the establish- 
ment of many new forms of generating income. One such concept that is widely used and distinctly different from regular employment is crowdworking.

\subsection{Crowdworking}

Crowdworking originated in the form of clickworking in the year 2000, when NASA was looking for a way to identify and categorize craters on Mars (Szpir 2002). This task required no scientific background, so NASA asked the general public for help. People volunteered and although they were not paid for their contributions they laid the groundwork for future crowdworking. Today the term crowdsourcing is often used, when a large group of people (a crowd) works on solving a problem, providing data or contributing to a common goal (Howe 2006; Brabham 2008; Doan et al. 2011). If the members of the crowd receive financial compensation for their work, one speaks of crowdworking (Durward et al. 2016; Blohm et al. 2013). Crowdworking becomes increasingly more popular and experts estimate that in 2013 there were 48 million registered crowdworkers of which about 4.8 million were active (Kuek et al. 2015). It is projected that by 2020 , crowdworking will generate gross services revenue in the range of $\$ 15$ billion to $\$ 25$ billion (Kuek et al. 2015).

The introduction of crowdworking, combined with the tense situation of the labour market, can have a significant impact on labour economics (Horton and Chilton 2010; Leimeister and Zogaj 2013). It gives many people the opportunity to become selfemployed, using crowdworking tasks and contracts as a base for their income. The advantages of this form of crowdworking are apparent: on the one hand, the firms have a huge pool of potential candidates to carry out their workload. In addition, they do not need to hire staff permanently, but can give as many contracts to the crowd as their current situation requires. On the other hand, there are also advantages for the crowdworkers. They can shape their work-life balance actively and can choose freely how many contracts they accept. They also benefit from all the other advantages of being self-employed, which can be a big motivation for crowdworkers (Kaufmann et al. 2011).

Of course, several problems arise in the context of crowdworking. Since the workers are all self-employed, there are no regulations on hourly wages. On average crowdworking platforms, crowdworkers earn less than 3 dollars an hour, while on platforms that require little qualification from their workers, the wages are even less (Rest 2015). So far, no regulations protect these workers from bad working conditions and crowdworkers have often no form of social security. Another challenge is the risk of wage dumping, i.e. the practice of paying lower wages than is usual in an industry (Astheimer 2015). This affects not only those directly involved in crowdworking, but all employees that work in a field where crowdsourcing is an viable option. These difficulties are of course more or less pronounced, depending on the specifics of the crowdworking variant, so it is necessary to differentiate between different types of crowdworking. The most common variants of crowdworking encountered today are described in the following section. 


\subsection{Variants of crowdworking}

There is a huge number of crowdworking platforms all around the globe. Since there are no binding rules and regulations, they all work differently. Most platforms specialize in one area, like content creation (copywriting, photography), programming or design. They all have in common that they offer a platform where people who are looking to participate in crowdworking can meet those who offer tasks and projects and are willing to pay for the fulfilment of this work. Nearly, all crowdworking platforms are financed by either a small percentage of all payments, which the platform keeps, a membership fee that workers or employers have to pay, or both.

The main difference between the platforms is the selection process, i.e. how it is decided which crowdworker gets which contract. There are also two distinct systems of how the wages are determined. Either the employers set the price for each task or the crowdworkers can choose the amount of money they want to receive as compensation for fulfilling the task. In order to precisely categorize crowdworking platforms, we define four categories.

Variant 1 is called "Cheapest Offer" and is similar to an auction. The employer gives specific information about the task, and crowdworkers can bid on it. After a certain time, the crowdworker with the cheapest offer gets the right to fulfil the task and in the end receives the agreed upon payment. It is obvious that this method favours workers with low reservation wages and is very susceptible to wage dumping, but the exact extend of these effects can only be quantified with further investigations. A popular platform that uses this method is freelancer.com.

Variant 2, termed "Quality Requirement", is similar to the auction that is held at "Cheapest Offer", but with the important difference that you need some sort of minimal qualification for each task. There are several ways of proving this qualification, for some platforms you need to maintain a certain success rate, other platforms require simply more experience within the platform, while some platforms charge their workers a fee for demonstrating their skill with online exams (Table 1).

Variant 3 is called "First Offer" and is a simple, but elegant system. Employers define the task and choose the offered payment. Then, the first worker who accepts this task is allowed to work on it. This system has the advantage of being extremely fast and simple to understand. It is most famously used by one of the biggest crowdworking platforms Amazon Mechanical Turk.

Variant 4 is termed "Best Quality" and focuses on the qualification of crowdworkers. All workers are allowed to bid for all the offered tasks, and the person best qualified gets the contract. A popular platform that uses this variant is topcoder. There are various ways how the qualification of the crowdworkers can be determined. One could look at past projects, reviews or ratings of finished contracts.

It is clear that all these variants of crowdworking have huge risks in terms of income security, working conditions and hourly wages, but the extend of these risks requires further investigation. Since it is difficult to obtain reliable data from the crowdworking platforms, the employers or the workers, an empirically calibrated, case-based study is not viable. Furthermore, we are especially interested in the differences caused by the used crowdworking method, which would only be discernible if we would have access 
Table 1 Matrix giving an overview of the considered variants of crowdworking

\begin{tabular}{lll}
\hline & Quality is irrelevant & Quality is relevant \\
\hline Payment set by worker & "Cheapest Offer" & "Quality Requirement" \\
Payment not set by worker & "First Offer" & "Best Quality" \\
\hline
\end{tabular}

to data from crowdworking platforms that use different variants, but are otherwise completely identical in terms of members, wages and offered contracts. Since this is not feasible with empirical data, we develop a computer simulation to study those systems more closely.

\section{Methods}

\subsection{Agent-based models}

In order to simulate the most important aspects of crowdworking, we use an agentbased computer model. Agent-based models (Gilbert 2008) are very successful in various fields, including but not limited to traffic simulations (Chen and Cheng 2010; Balmer et al. 2004; Doniec et al. 2008; Hofer et al. 2018), city planning (Batty 2007), evacuation models (Chen and Zhan 2008) or complexity research (Axelrod 1997). A main application of agent-based models are all systems in which human behaviour and decision-making are important components, e.g. social science (Davidsson 2002; Epstein 1999) or human systems (Bonabeau 2002). Also the field of agent-based computational economics (Amman et al. 1996) is quite active (Tesfatsion 2002, 2006) and provides a valuable contribution to the economic discussion (Farmer and Foley 2009). It is possible to simulate whole economies (Deissenberg et al. 2008) and the heterogeneity of individuals can be incorporated elegantly (Hommes 2006).

The goal of each agent-based model is to simulate a system not from the top down, but from the bottom up, in our case by starting from the individuals who want to participate in crowdworking. This approach has many advantages. First of all, we do not have to make assumptions on the whole system, but our starting point is an accurate, yet abstract description of the agents, i.e. the workers. All other phenomena then emerge from the interaction of the agents with each other and with their environment, i.e. the crowdworking platform. This property, that phenomena arise in a simulation, even though they cannot be predicted from the rules and the behaviour of the individual agents, is called emergence, and a common property among agent-based models (Dessalles et al. 2008). However, emergence within an agent-based model does not necessarily mean that this phenomenon can also be observed in reality (Boero and Squazzoni 2005; Epstein 2006).

Another big advantage of agent-based models is that human behaviour can be incorporated in an elegant manner (Bankes 2002; Axtell 2000). Although it might be difficult to find exact equations on when workers accept a contract and when they decline for example, finding simple rules that approximate their behaviour is 
sufficient to construct an agent-based model. Additionally, this allows us to deal with uncertainties. Even though we are not aware of, for example, specific reservation wages of each individual agent, we can distribute this property randomly with a reasonable distribution to account for the heterogeneity of the population. Agent-based models also allow for a high level of abstraction, which makes the obtained result independent of specific details of the economy, the overall labour market or nation-specific rules and regulations.

Thanks to those advantages, especially in the context of labour and labour market research, agent-based models proofed to be viable tools for answering various research questions. For macroeconomic policy design, agent-based models can be used as laboratories to test the policy effects on macrodynamics (Fagiolo and Roventini 2017). An artificial, virtual labour market can be simulated with an agent-based approach (Neugart et al. 2012; Chaturvedi et al. 2005), but also more specialized models are possible. Labour supply can be investigated (Leombruni and Richiardi 2006) as well as labour migration (Naivinit et al. 2010). Agent-based models can also be used to approximate the effects of labour market reforms upon unemployment and income inequalities (Dosi et al. 2016). Other examples can be found in Dawid et al. (2012, 2014), where an agent-based model is applied to analyse the convergence of regions characterized by local labour markets and heterogeneous skills. All these areas benefit from the bottom-up approach, in which we only need to understand the behaviour of the agents, i.e. primarily the employers and the employees to generate a model and find non-trivial, aggregated results on the macro-scale.

\subsection{The model}

The purpose of this agent-based model is to compare different variants of crowdworking in a general way, so that the obtained results are independent of specific details of the crowdworking platform. It features many adjustable parameters that can be used to calibrate the model to empirical data, but also when not calibrated it yields essential results about crowdworking in general. The model is available for download on OpenABM. ${ }^{1}$

\subsubsection{Overview}

Agents compete for contracts on a virtual crowdworking platform. Each agent is defined by various properties like qualification and reservation wage. Agents that are unable to turn a profit have a chance to quit the crowdworking platform, and new crowdworkers can replace them. Thus, the model has features of an evolutionary process, filtering out the ill-suited agents and generating a realistic distribution of agents from an initially random one. To simulate a stable system, the amount of contracts issued per day can be set constant, as well as the number of crowdworkers. If one is interested in a dynamically changing platform, the simulation can also be initialized in a way that increases or decreases the number of crowdworkers or number of contracts over time. Thus, a large variety of scenarios can be investigated.

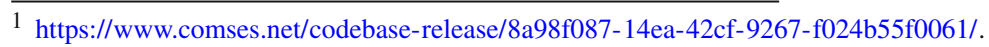




\subsubsection{Agents}

Of course the agents are the most crucial part of such a model . They are defined by various properties that are distributed heterogeneously. Each agent has an individual cost of living, which measures the expenses each agent has each month. The average of this cost of living is 1000 monetary units (MU) with a standard deviation of $100 \mathrm{MU}$, meaning that more than $95 \%$ of the agents have a cost of living between 800 and 1200 MU. In addition, each agent has a certain qualification. Qualification defines the quality of work an agent can produce. Qualification is uniformly distributed between 0 and 10, and as such is an abstract measure of qualification and not directly related to education. A qualification value of 9.5 just means that the agent is better qualified than $95 \%$ of its competitors. Each agent has a reservation wage. It is based on the average payment received for a contract of the agents qualification level; thus, higher qualified agents have a higher reservation wage. The additional payment for each level of qualification is a parameter that can be chosen freely.

These agents interact with a virtual crowdworking platform. This platform is abstracted in the sense that it only contains contracts with various properties, but no individual employers, corporations or firms. All contracts have a required quality, which represents the qualification of the workers that the clients desire and an expected cost, which represent the amount of money the clients are willing to pay for this contract. The required quality is distributed uniformly between 0 and 10 to match the qualification of the workforce. The interpretation is the same, a contract with a required quality of 1.5 means that $15 \%$ of the crowdworkers will be not qualified enough for this contract. Each contract also has an expected cost, which is the average reservation wage of an agent with exactly the right qualification level.

\subsubsection{Adjustable parameters}

Because of its abstract approach, the presented model has many parameters that can be chosen freely in order to simulate different scenarios. It is also possible to calibrate the model to empirical values or show that some results are independent of the specifics of those parameters. Table 2 shows all the adjustable parameters and their default values. For a detailed analysis of the impact of the most important parameters, see Sect. 3.4.

The default values were selected as follows. One MU is defined as the average cost of living divided by 1000; thus, the average cost of living is $1000 \mathrm{MU}$. In order to simulate a stable crowdworking platform, in which it would in theory be possible for every worker to earn a decent living wage, 1000 workers compete for 500 contracts a day, which pay at least $150 \mathrm{MU}$. Thus, on average a crowdworker earns $2250 \mathrm{MU}$ a month, leading to an average possible profit of more than $1000 \mathrm{MU}$ for each worker. For each level of qualification, the payment increases by $5 \mathrm{MU}$, so that the payment for contracts ranges from 150 to $200 \mathrm{MU}$, i.e. the more difficult contracts pay a third more than the simpler contracts. This value is consistent with the recommended paying rates on prominent crowdworking platforms. The chance to quit after a month of negative profit is derived from the idea that crowdworkers have to look for alternatives if their savings are depleted. Most financial advisers recommend a liquidity (i.e. the relation between savings and monthly expenses) of 2-6 (DeVaney 1997), i.e. enough savings 
Table 2 Adjustable parameters of the model

\begin{tabular}{lll}
\hline Parameter & Description & Default value \\
\hline Workforce & The number of crowdworkers active on the platform & 1000 \\
Contracts & The number of contracts issued every day & 500 \\
d-workforce & The change of the number of crowdworkers per month & 0 \\
d-contracts & $\begin{array}{l}\text { The change of number of contracts issued every day per } \\
\text { month }\end{array}$ & 0 \\
Payment & $\begin{array}{l}\text { The minimal payment for a one-day contract } \\
\text { Payment-factor }\end{array}$ & The additional payment for a one-day contract for each \\
level of qualification & 5 MU \\
Competition & The percentage of workers with a significantly lower & $0 \%$ \\
cost of living & The relative reduction of cost of living and reservation \\
Competition-factor & The chance of a worker to quit after a month of negative & 0.5 \\
puit-chance & profit & $25 \%$ \\
\end{tabular}

to cover 2-6 months without income. With a quit-chance of $25 \%$ also in the model, the workers have to quit after 4 months without profits on average.

\subsubsection{The simulation}

In order to obtain quantitative results for different indicators, we simulate this system of workers and contracts over a long period of time. In the simulation, time is discretized into units of $10 \mathrm{~min}$, which is fine enough to observe all important effects, yet coarse enough to lead to a reasonable computation time for each simulation run. For this study, one run simulates 3 years of crowdworking, the first year is done so the market has time to find a balance so that the initially random population of agents has no influence on the results. Then, over the course of the next 2 years, the relevant data are extracted each month.

A single simulation run is performed as follows: the system is initialized with the chosen population of crowdworkers. They are randomly distributed on a 101 times 101 grid symbolizing the crowdworking platform. This grid represents informational frictions: crowdworkers need time to look for suitable contracts. In each time step, there is a chance that a new contract is generated on a random position on the grid. All agents that are currently not working on a contract look for a new contract by moving to a neighbouring position on the grid. If they find a contract there, they place an offer consisting of their own qualification and their own reservation wage. After this bid, they continue looking for more contracts. In that way, each contract gets many offers. 24 hours after the contract was added to the platform, the best offer is selected. The criteria for deciding which offer is the best depend on the selected variant of crowdworking, detailed in the next section. The agent with the winning offer now stops searching for more contracts and has to invest its time in fulfilling the contract in order to receive the agreed upon monetary compensation. The fulfilled contract 
Fig. 1 Decision process of a crowdworking agent

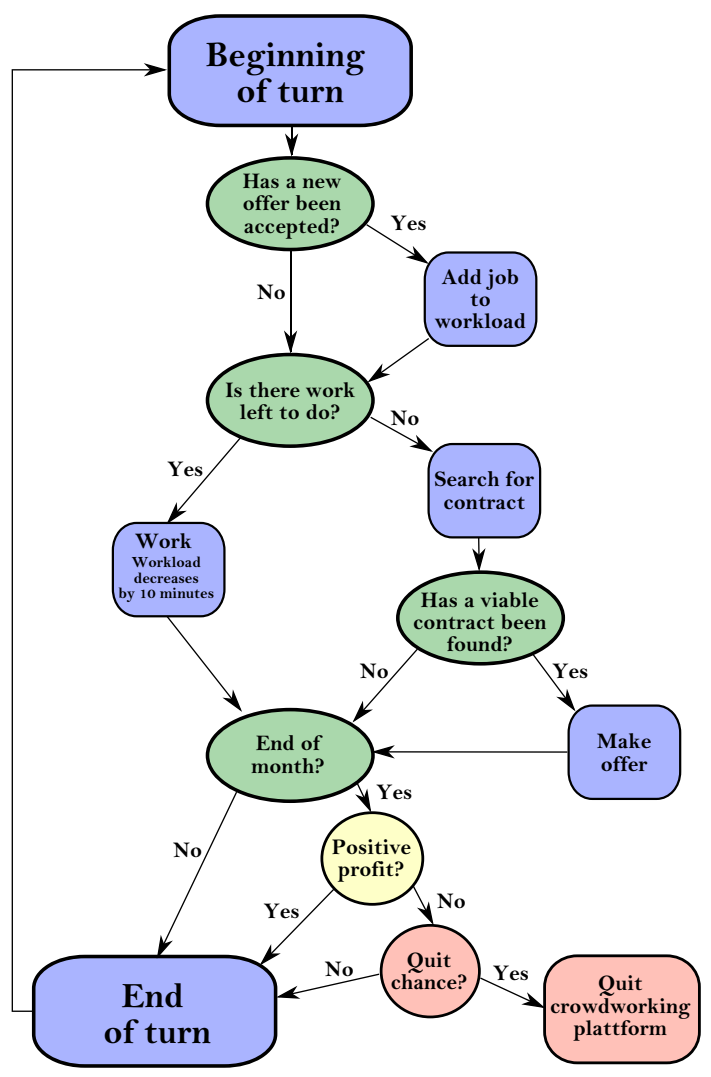

disappears. There is no maximum of contracts that a single worker can accumulate. Thus, they bid on every contract that is relevant to them and only stop searching for offers if they are actively working on fulfilling a contract.

Once a month all agents calculate their profit by subtracting their monthly cost of living from their earnings. Agents with negative profit have a certain chance to leave the platform and need to look for different ways to cover their cost of living. In order to investigate a stable system, new random agents are generated each month to replace those leaving the system. Note that it is also possible to investigate more dynamic systems, in which the number of agents or the number of daily contracts can change each month. However, here we are mainly interested in the situation of crowdworkers in an stable system. Therefore, we do not change these parameters over time. An overview of how an agent acts each turn is given in Fig. 1.

Even with a stable number of agents this simulation leads to an evolutionary process: workers that are unfit to earn enough money to cover their cost of living will soon quit the platform and a different, random agent replaces it. Depending on the specifics of how contracts are given out, this could favour highly qualified workers or workers with a small reservation wage. In turn, this change in composition of the agent population influences the competition. After a few months, some form of balance is reached and 
Table 3 Qualification levels

\begin{tabular}{ll}
\hline Qualification level & Range \\
\hline Very low & $q<2$ \\
Low & $2 \leq q<4$ \\
Medium & $4 \leq q<6$ \\
High & $6 \leq q<8$ \\
Very high & $8 \leq q$ \\
\hline
\end{tabular}

investigating this balance and the relation to the variant of crowdworking is one of the key findings of this study. Therefore, also the initial Gaussian distribution of many agent properties is not so important for the final result, since its biggest influence is during the equilibration phase. After equilibration, the distribution of agents can be completely different from the initial distribution, as can be seen in the result section.

There are four key aspects that we calculate during the simulation. The most important one is the utility function $u$ of each agent. In this simplified model is it calculated as a profit $p$, i.e. the amount of money $e$ they earn during a month minus the cost of living $c$. For the agent with index $i$, this leads to

$$
u_{i}=p_{i}=e_{i}-c_{i}
$$

Limiting the utility function to a simple profit has a few advantages. Primarily we can use the obtained profit directly to judge whether an agent was successful or not, by checking if it has a positive profit or not. Thus, we will limit our investigation of the utility function of an agent to its profit.

In addition, we also count the number of agents for each qualification level in order to gain insight into the distribution of the qualification of the workers, and therefore, the quality that is delivered. Since qualification is real-valued, we bin the qualification level into the categories given in Table 3 .

The third property we calculate relates to job security and the risk of being forced to quit crowdworking because of too small earnings. Every month we count the agents that turned a negative profit. Thus, we gain insight into the relative chance to loose one's job per month. Related to this property is the percentage of workers with low income. For this property, we do not count the workers with negative profit but only those with positive profit smaller than 1000 MU. Those workers will not quit the crowdworking platform, but they are at the risk of becoming working poor.

These properties give a good overview over the most important aspects of a crowdworking system. Naturally, the results are very sensitive to the way the contracts are distributed. Since there are several competing ideas on how this distribution of work is done, we investigate the four most common variants and compare them. The implementation of these different variants is outlined in the following section.

\subsection{Implementation of different crowdworking variants}

We implemented four different variants how the "best" applicant for each contract is chosen: 
- Variant 1: "Cheapest Offer", where the applicant with the lowest reservation wage is selected

- Variant 2: "Quality Requirement”, where the cheapest offer with a minimal quality is chosen

- Variant 3: "First Offer", where the first worker who applies for the contract is selected

- Variant 4: "Best Quality", where the applicant with the highest qualification level is chosen.

For variant 1 "Cheapest Offer", all applicants can apply for all contracts. They only give their reservation wages; other properties like their qualification or the expected quality of the contract are ignored. After a certain time, all applicants are sorted by their reservation wage. The applicant with the lowest offer is selected.

In variant 2 "Quality Requirement”, the process is similar; however, only workers with a qualification level that is at least equal to the expected quality of the job are allowed to apply. From these applicants, the one with the lowest offer is selected.

Variant 3 "First Offer" ignores the income expectations of the worker as well as any qualification or expected quality. The employer sets the price for the contract, and the first worker to accept this offer is selected.

For variant 4 "Best Quality", all applicants are allowed to apply for the contracts. However, the employer sets the price and selects the applicant with the highest qualification.

In order to gain insight into the effect of globalization on these different scenarios, all variants are also simulated in the context of international competition. In these versions, a certain percentage of the workers are international competitors, who live in a region with significantly lower cost of living. The cost of living and therefore the reservation wages of such workers are distributed similarly to national workers, but multiplied with a factor $<1$. Of course this constitutes a huge simplification of a real crowdworking platform, where workers from many different nations compete, but even in this approximation the most basic effects of globalization should be visible, i.e. the direct effect of workers with different cost of living competing for the same contracts.

\section{Results}

\subsection{Profit for crowdworkers}

In order to investigate the differences between the distinct variants of crowdworking, the simulation is performed for each variant using the default parameters given in Table 2. Data are gathered each month for a period of 2 years after an equilibration phase of 1 year. Figure 2 shows the profit distribution of the crowdworkers in the form of violin plots. The thickness of the shape in $x$ direction shows the relative amount of crowdworkers with the profit given on the $y$ axis. The horizontal lines show maximum, average and minimum. Different colours indicate different qualification levels. 

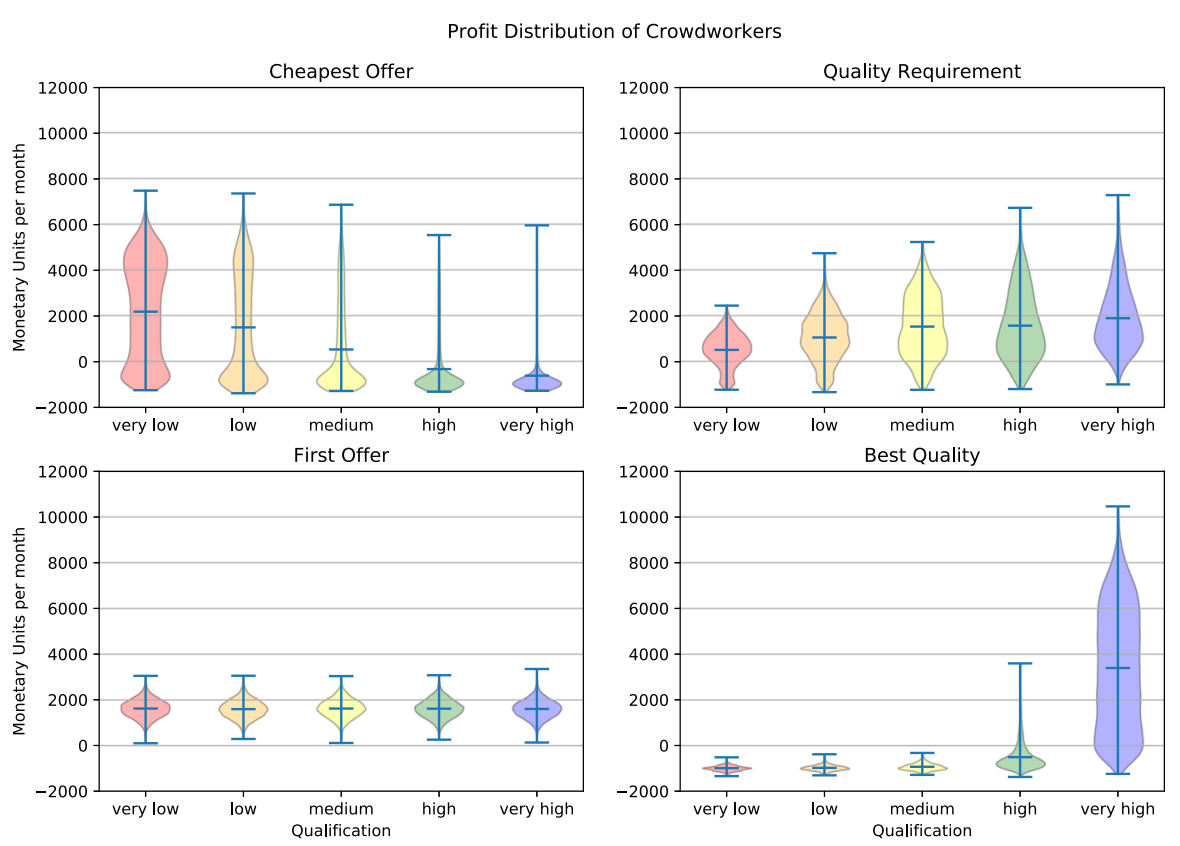

Fig. 2 Profit distribution of workers with very low (red), low (orange), medium (yellow), high (green) and very high (blue) qualification. Each quadrant shows a different variant of crowdworking (color figure online)

For variant 1 "Cheapest Offer", it can be seen that the highest profit can be achieved by the workers with the lowest qualification. They have low reservation wages, and thus, are able to secure more contracts than their better qualified competitors. On average, they make a profit of more than 2000 MU each month. The distribution also shows a maximum around $4250 \mathrm{MU}$, which corresponds to an agent working nearly constantly on contracts and has an average cost of living. Thus, we know that some of the workers in this qualification class are at their maximal contract capacity. For better qualified agents, this maximum is not visible any longer. Average profit decreases for the classes with higher qualification, the last two classes have a negative average profit. Negative profits also occur for the other classes, with a maximum at $-1000 \mathrm{MU}$, which is the average cost of living. Thus, these agents were unable to secure a single contract and will probably leave the crowdworking platform soon.

For variant 2 "Quality Requirement", the distribution of profits is reversed, when compared to variant 1 . Here, the agents with the highest qualification have the highest average profit. They can apply for nearly all contracts, which gives them a significant advantage and they can secure much more contracts. However, no class has a negative average income. Since the number of contracts in each variant is the same, this means that also the maximal profit is lower than in variant 1 . Profits are distributed in a way that resembles a Gaussian distribution, with some agents having a deficit in every qualification class.

For variant 3 "First Offer", the profit distribution looks completely different. Since the process of selecting the best offer is not affected by the qualification of a worker or 

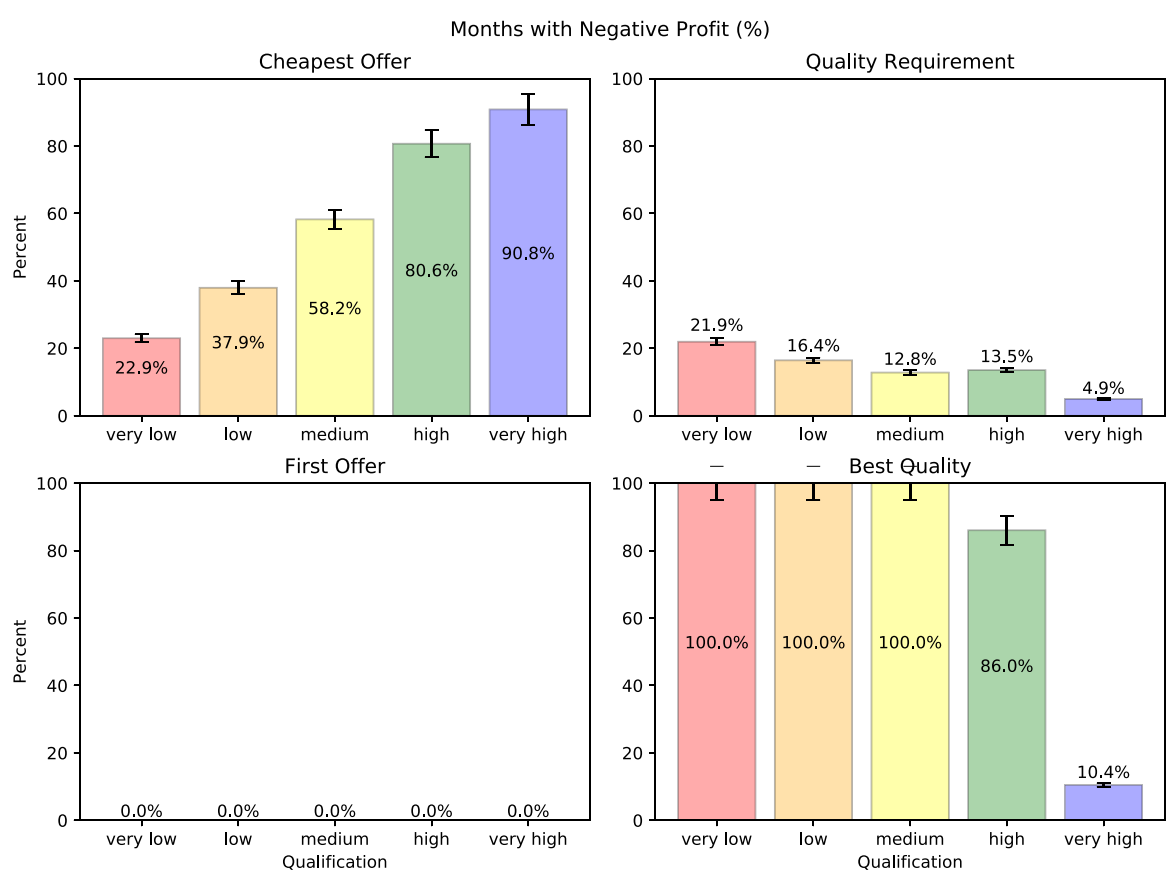

Fig. 3 Percentage of months with negative profit for the different qualification levels. Each quadrant shows a different variant of crowdworking

their reservation wage, the profit is the same for all qualification levels. It is distributed Gaussian with a mean of roughly 1500 MU. No worker experiences a negative profit; however, there are also only a few workers that generate a profit of more than $2000 \mathrm{MU}$ per month.

Variant 4 "Best Quality" shows a rather extreme profit distribution. All but one qualification level shows negative profit on average. Only workers of very high qualification are able to turn a significant profit. This variant also leads to the highest profit of all variants, with some agents generating more than $8000 \mathrm{MU}$ a month.

This shows that even if the crowdworking platform offers the same contracts, the variant of crowdworking has a huge impact on the resulting workforce and their success in earning a living wage. "Cheapest Offer" attracts low quality workers. Because of the possibility to secure more contracts than the competition by requesting smaller wages, average wages are relatively small. In the variants "Quality Requirement" and "Best Quality", it is more difficult to succeed for workers with low qualification, but average wages are higher. Since quality is more important here than reservation wages, wage dumping is a less successful strategy.

If we look at the profit distribution in more detail, we can derive further properties of the crowdworking system. Job security is one of the most important ones. It tells us how many crowdworkers have to quit because they are unable to earn enough. Job security can be related to the percentage of months that yielded a negative profit for a crowdworker. This percentage is depicted in Fig. 3. 
For variant 1 "Cheapest Offer", job security is relatively low. For workers with the lowest qualification roughly $23 \%$ of all months yield negative profit. This number increases with qualification, up to over $90 \%$ for very highly qualified workers, meaning most of them will not be able to succeed in this variant.

In variant 2 "Quality Requirement", job security is much better. The workers with the lowest qualification have negative profit in about $22 \%$ of all months. For all other qualification classes, this value is lower. For very highly qualified workers, it is roughly $5 \%$.

An interesting effect can be observed for variant 3 "First Offer". Since qualification and reservation wage have no influence on the distribution of contracts, all workers have similar earnings and thus similar profit. If there is enough money in the system for all workers to earn a profit, as is the case for the default parameters of the model, there are no months with negative profits in any of the qualification classes.

The worst job security can be seen for variant 4 "Best Quality". Workers of medium or lower qualification have no months with positive profits at all, and also those with high qualification have $86 \%$ months with negative profit. Only the workers with very high qualification have a significant chance to remain in the crowdworking platform.

We see that also job security is highly affected by the variant of crowdworking used. Especially the variant "Cheapest Offer" is extremely risky. Due to the possibility of wage dumping and therefore and inadvertent selection process that favours agents with lower reservation wages, even the most successful qualification class has less job security than any class in the variants "Quality Requirement" and "First Offer".

The percentage of months with negative profit is a good indicator for job security, yet having to quit crowdworking because of a lack of earnings is not the only risk crowdworkers face. Also exploitation, self-exploitation and working poor are possible consequences of crowdworking that need to be investigated. Although the model does not give any insight into these phenomena, we can at least assume that workers with small profit are most prone to those issues. Figure 4 shows the percentage of months with low profit for the different qualification levels. The definition of "low" income is in this case arbitrary, and we defined it as a profit below $1000 \mathrm{MU}$, which allows to compare the different variants of crowdworking.

For variant 1 "Cheapest Offer", the percentage of low income months is similar for the qualification levels very low up to medium with roughly $10 \%$. Highly and very highly qualified workers have less months with lower income, mainly because they have higher reservation wages on average, meaning they either make no profit, because they cannot get any contracts or a significant profit, because they request higher payment than their lower qualified competition.

In variant 2 "Quality Requirement", there are a high fraction of low profit months, especially for workers with very low qualification. The main reason for this effect is that those workers can only apply for a small fraction of the contracts that are on the platform. So even though their offer is likely to be accepted because of their low reservation wage, the low number of contracts accessible to them leads to a low profit overall.

For variant 3 "First Offer", all qualification levels are very similar and the number of months with low income is the same (within error bars) for every qualification level. It is roughly $6 \%$, so lower than the average value for all other crowdworking variants. 


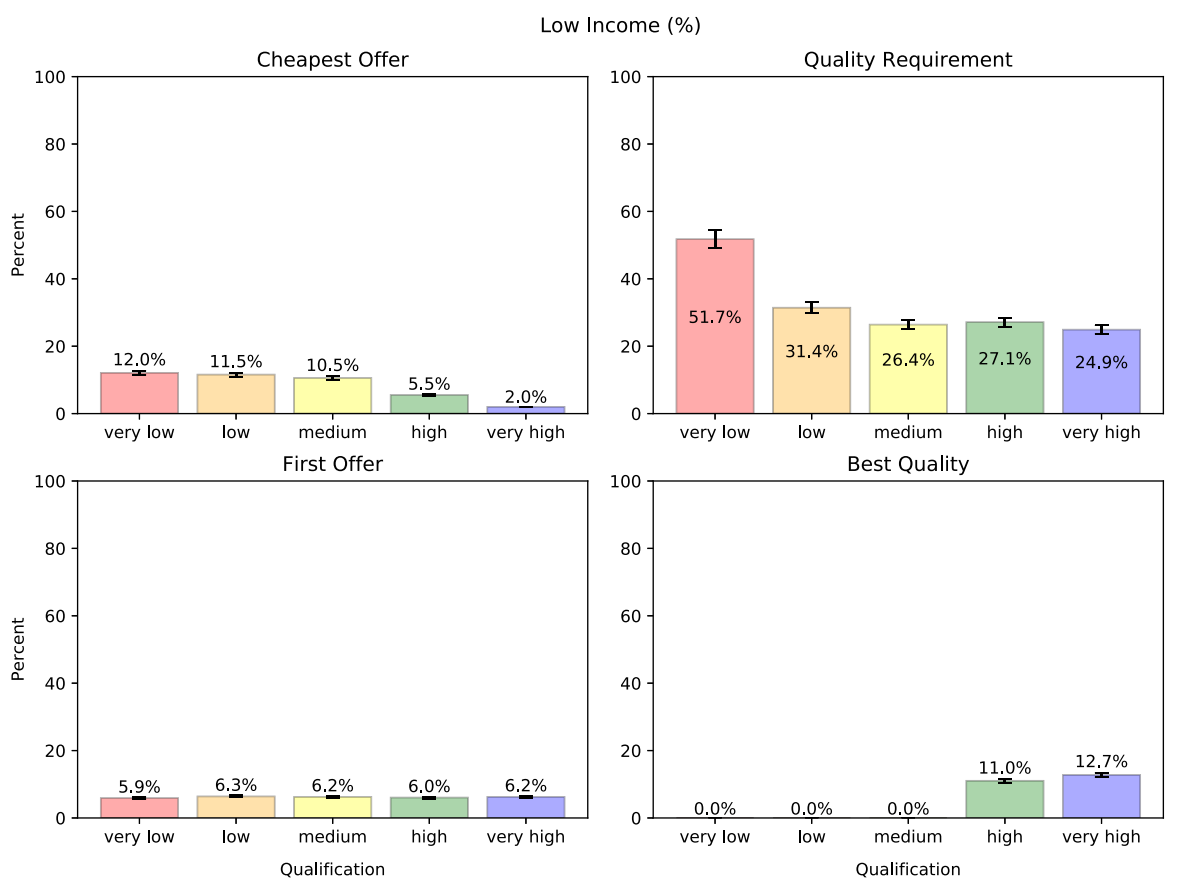

Fig. 4 Percentage of months with low profit $(<1000 \mathrm{MU})$ for the different qualification levels. Each quadrant shows a different variant of crowdworking

Considering that there are also no months with negative profit in this variant, one can say that income is indeed evenly distributed among the crowdworkers.

Variant 4 "Best Quality" has no low profit for the qualification levels up to medium, because there are no workers with these qualification who make any positive profit. Positive profit is only possible for highly and very highly qualified workers. Here, the amount of low profit months is $11 \%$ and $13 \%$, respectively.

With respect to low income months, the variant "Quality Requirement" might be the most problematic. The income is distributed among a lot of workers and since both workers with high reservation wages and workers with low qualification struggle to secure a lot of contracts, many of them earn very little, compared to the other variants.

\subsection{Workforce composition and delivered quality}

An interesting effect that can be observed with the presented model is the evolutionary process that filters out agents that are not well suited to compete on the crowdworking market. This leads to stable states with a non-trivial distribution of agents that can be very distinct from the initial population. This of course also influences the average delivered quality and is as such an important indicator from an employer perspective. The agent distribution is highly dependent on the variant of crowdworking and is depicted in Fig. 5.

For variant 1 "Cheapest Offer", a large fraction of the workforce (nearly 60\%) are of very low or low qualification. Better qualified workers are rarer. This is caused by 


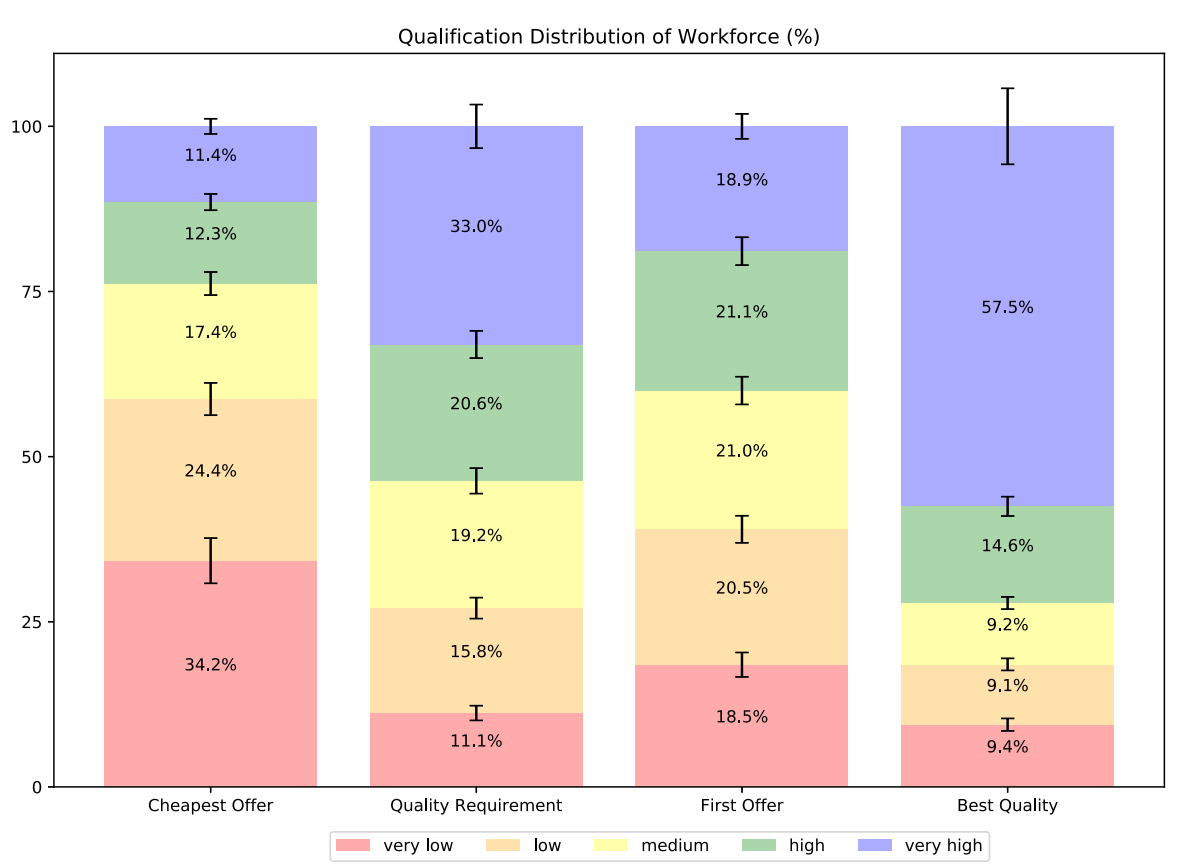

Fig.5 Qualification distribution of the workforce in balance

the fact that lesser qualified workers are able to secure more contracts and stay in the market, while workers with higher reservation wages are often forced to quit because they are unable to find enough contracts.

In variant 2 "Quality Requirement", the majority (more than 50\%) of the workers are of high or very high qualification. Workers with lower qualification have access to less contracts, and it is therefore more difficult for them to acquire enough contracts.

The distribution in variant 3 "First Offer" is nearly uniform. Each qualification group comprises roughly $20 \%$ of the workforce. Since the qualification and reservation wage has no influence on the number of acquired contracts, no group has an advantage over the others.

The largest skewness in qualification can be observed in variant 4 "Best Quality". Here, nearly $60 \%$ of the workforce are very highly qualified. In this variant, only the workers who produce the highest quality of work are selected for a contract, all others are filtered out rigorously. There is no way to compensate with lower reservation wage, so only highly qualified workers can generate a profit in this system, leading to this one-sided distribution.

\subsection{Effect of competition from crowdworkers with lower cost of living}

An important phenomenon on crowdworking platforms is the effect of globalization, since often workers from different countries with different cost of living, and thus, reservation wages compete for the same contracts. While this is also true for any labour 

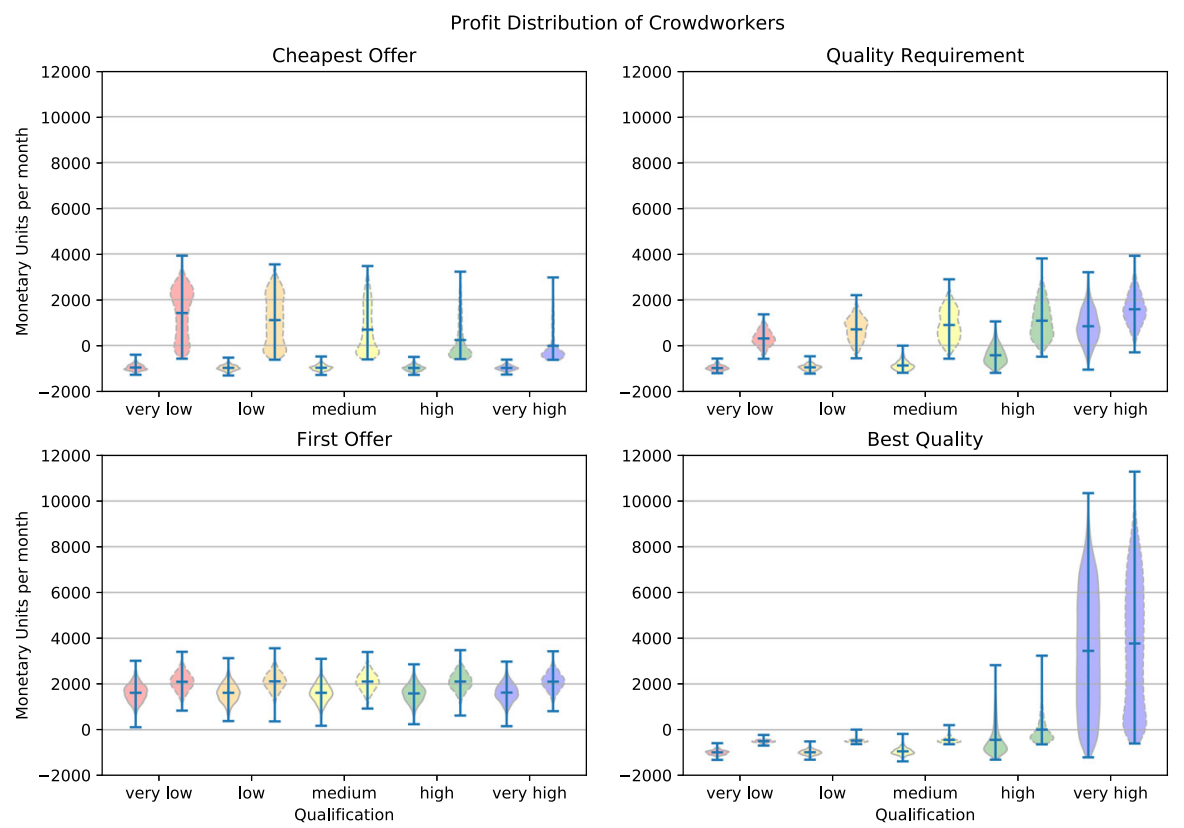

Fig. 6 Profit distribution of workers with very low (red), low (orange), medium (yellow), high (green) and very high (blue) qualification. The left distributions are for workers with the normal cost of living, the right distributions (with the dashed outline) are for the workers with reduced cost of living. Each quadrant shows a different variant of crowdworking (color figure online)

market in which workers from different countries are in direct or indirect competition, for crowdworking the impact of globalization can be observed rather quickly, since it is a highly dynamic market that can react swiftly to changes because of a lack of long-term contracts. Furthermore, geographical distance between employer and employee plays no role on a crowdworking platform. To gain insight into such form of competition and the way it affects the different crowdworking variants we add international competition to the model. Half of the workforce consists of workers from a different country with a lower cost of living thus a lower reservation wage. The presented simulation runs use a decrease factor of 0.5 , meaning the reservation wage and cost of living is halved. The income distribution that arises is shown in Fig. 6.

The influence of international competition is most drastic for variant 1 "Cheapest Offer". Here, none of the workers with high reservation wages are able to secure any contracts and the market is completely dominated by low income workers. Their income distribution is similar to the one shown in Fig. 2, i.e. a higher profit for lower qualified workers. However, since the number of contracts issued per day is the same and the number of suitable workers is effectively halved, average income is roughly doubled. Thus, this variant of crowdworking massively favours workers with lower reservation wages.

In variant 2 "Quality Requirement", the situation is similar. Workers with lower reservation wages are able to secure more contracts. However, the effect is less pronounced than in variant 1 . Some highly and most of the very highly qualified workers 
are able to turn a profit, even if their reservation wage is high. For such contracts, there is less competition, so the workers with high reservation wages are able to secure some contracts. Although they get less contracts, they receive more payment per fulfilled contract which compensates this disadvantage. The average profit of highly qualified workers is similar for national and international workers. With the exception of the highest qualification class, this variant of crowdworking significantly favours workers with lower reservation wages.

In variant 3 "First Offer", the situation is different. Both national and international workers are able to generate profit. Since reservation wages and qualification have no influence on the number of obtained contracts and payment, the only difference in profit is caused by a different cost of living. While all workers earn roughly the same, international workers have lower expenses. However, the actual distribution of contracts has no bias for or against international workers, so this system does not favour one group intrinsically, in contrast to variant 1 and variant 2.

Variant 4 "Best Quality" is similarly unbiased. The main property that determines whether a worker gets a contract is their qualification, irrespective of their reservation wage and cost of living. There is not much difference between the same variant without international competitors: Very low, low and medium quality workers, national as well as international, do not get many contracts and are unable to turn a profit. The very highly qualified workers earn high profits. The difference between international and national competitors is only caused by the different cost of living. Therefore, this system has a huge bias towards high quality workers, but does not favour workers with higher or lower reservation wages intrinsically.

We see that not all variants of crowdworking behave in the same way when introducing international competitors. While variant 1 and variant 2 make it nearly impossible for workers with higher reservation wages to cover their cost of living, variant 3 and variant 4 offer no big advantage for international workers. The only difference in profits here is directly caused by the smaller expenses of international workers.

The second interesting characteristic, which is sensitive to international competition, is job security. Again we look at the percentage of months with negative profit in more detail to find a suitable indicator for job security. Results of this investigation are presented in Fig. 7.

For variant 1 "Cheapest Offer", the job security is extremely biased. Workers with high reservation wage never turn a profit, but also the highly qualified international workers have great difficulties staying in the market. For the international workers, job security is better than for national workers without international competition, because the national workers are no real competition and the number of contracts per worker effectively doubles.

In variant 2 "Quality Requirement", job security is high for workers with low reservation wages as well as for very highly qualified crowdworkers. Other workers cannot cover their cost of living and are filtered out.

No effect regarding job security can be observed for variant 3 "First Offer". Even with international competition with significantly lower reservation wages and cost of living, all workers are able to turn a profit.

Also for variant 4 "Best Quality", international competition has no significant effect on job security. Very highly qualified workers have good job security, while all others 

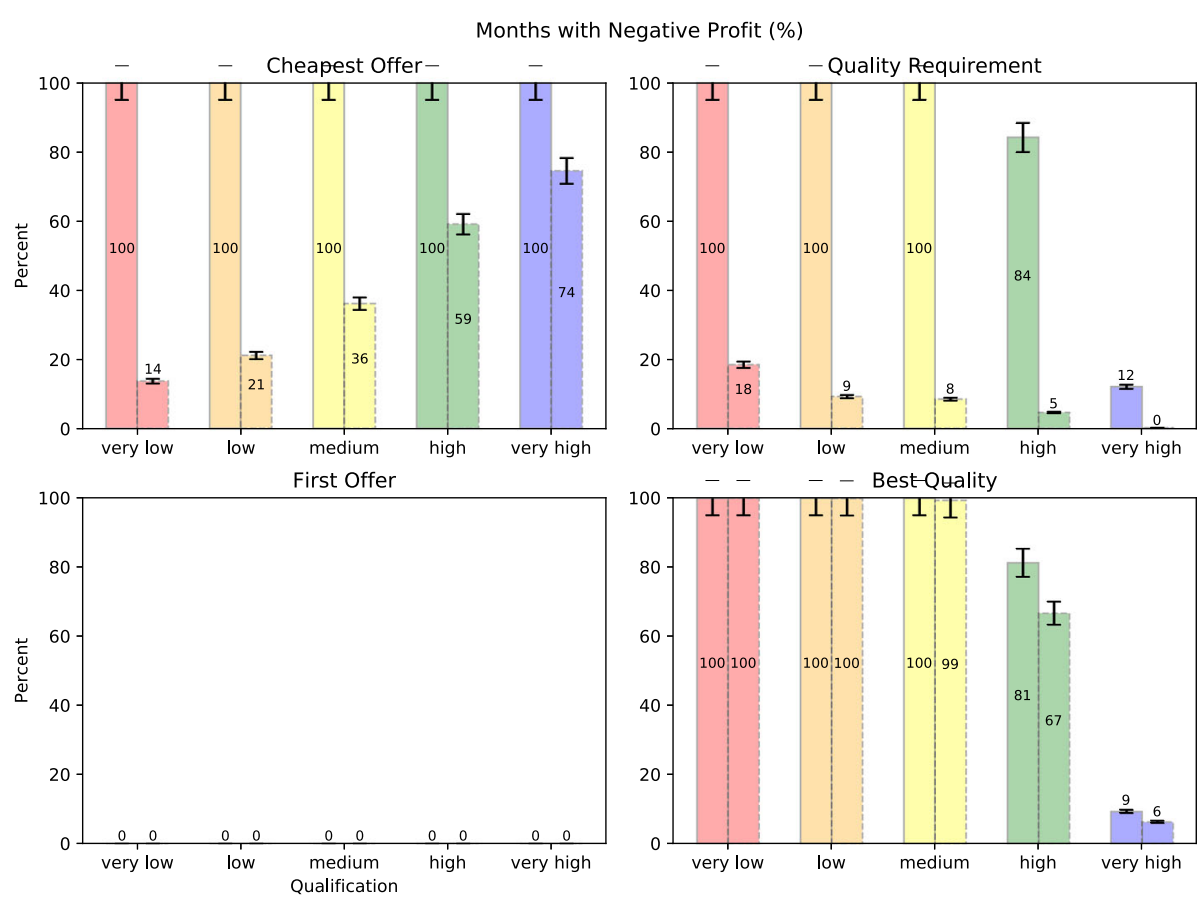

Fig. 7 Percentage of months with negative profit for the different qualification levels. The left bars are the workers with normal cost of living, the right bars (with the dashed outline) are the workers with reduced cost of living. Each quadrant shows a different variant of crowdworking

have great difficulties turning a profit. The small differences between national and international crowdworkers are caused by their lower cost of living.

Looking at job security substantiates the previous insight, that all variants of crowd working in which the crowdworkers can choose their payment themselves favour competitors with lower cost of living. This bias is especially strong in variant 1 "Cheapest Offer", where national competitors are unable to generate profit, no matter their qualification. Variant 3 and variant 4 are nearly unbiased in terms of reservation wage. The only differences in profit are caused by a different cost of living.

\subsection{Sensitivity analysis}

We took great care in designing a model that is universally applicable and independent of specifics of the labour market or the economy. Nevertheless, there are still many exogenously defined parameters (see Table 2) with significant influence on the results. Those need to be put in from outside the model, depending on various specifics of the investigated system. To show the influence of these parameters on the obtained results, we perform a sensitivity analysis. The results of this analysis are presented in the following figures.

Figure 8 shows the effect of changing the number of contracts issued per day. We investigate contract-worker ratios ranging from 0.3 to 0.7 . For variant 1 "Cheapest 
Sensitivity to available contracts per day
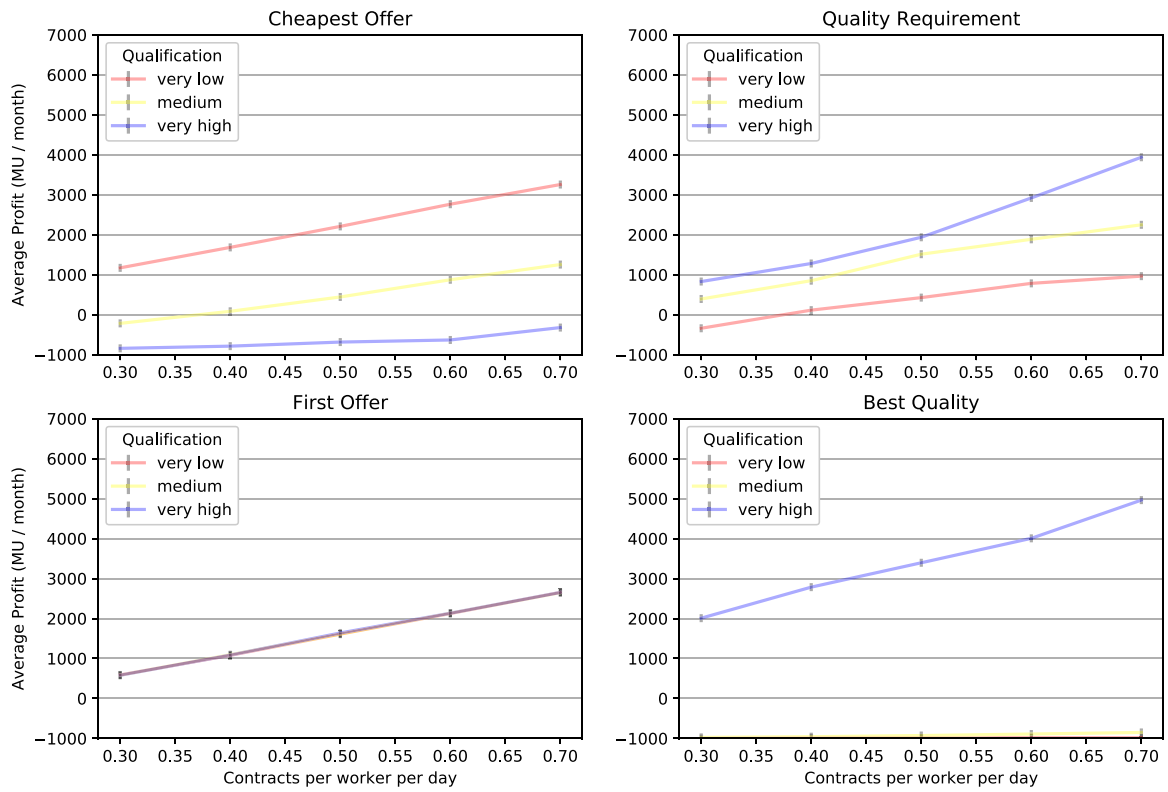

Fig. 8 Sensitivity analysis: the effect of the available contracts per day
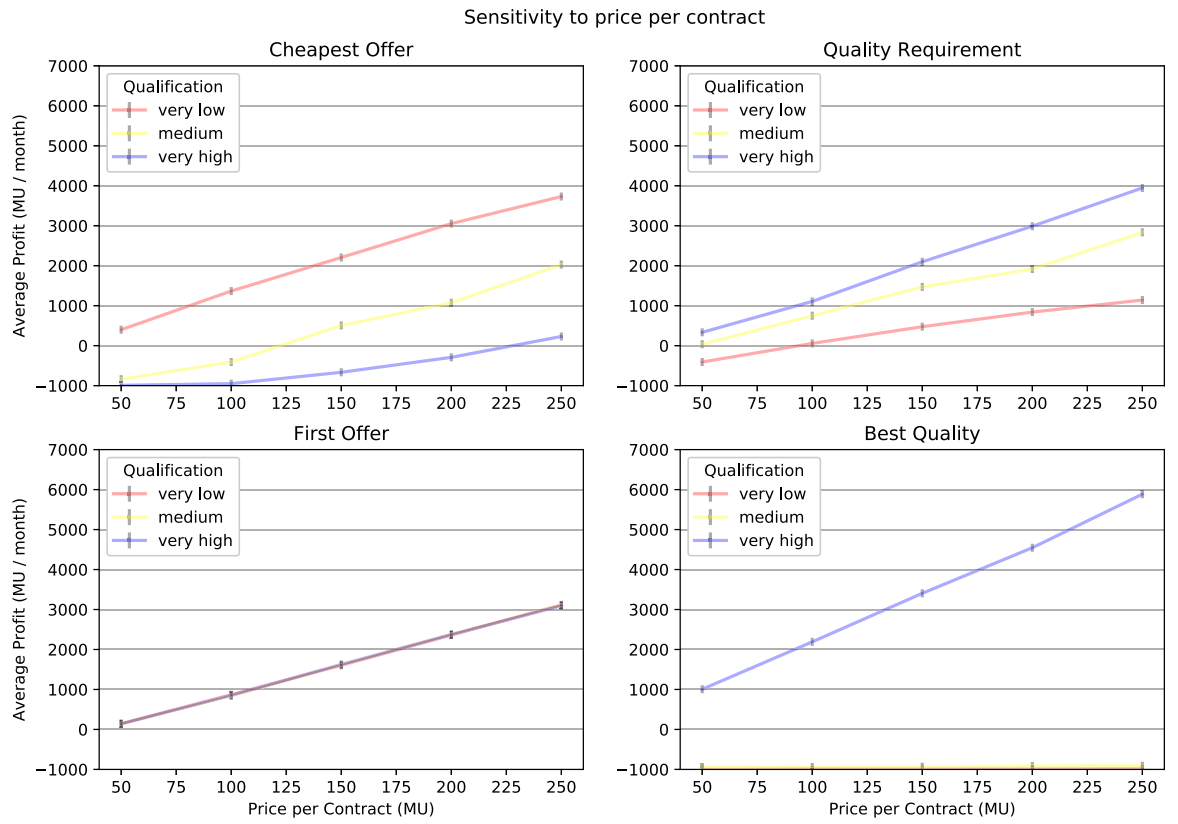

Fig. 9 Sensitivity analysis: the effect of payment per contract 
Sensitivity to price difference between qualifications
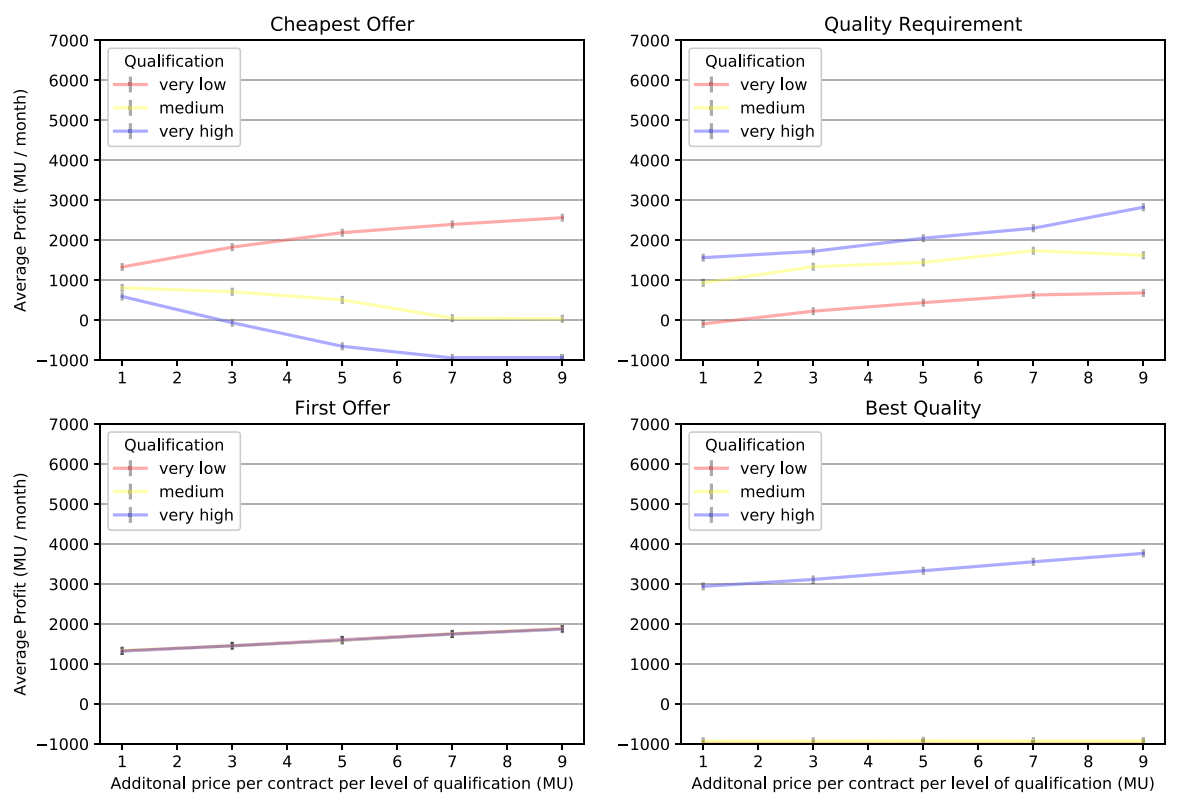

Fig. 10 Sensitivity analysis: the effect of the additional payment per level of qualification

Offer", the main finding was that workers with lower qualification have a higher profit on average. This property is maintained across the investigated range of the parameter, although average profit naturally increases with additional contracts. For variant 2 "Quality Requirement", the situation was reversed: highly qualified workers had the highest profit. Also this observation is independent of the number of contracts per day. Variant 3 "First Offer" showed no differences between the qualification levels. Even if the number of available contracts is changed, this property is still maintained. In variant 3 "Best Quality", only the very highly qualified workers were able to turn a profit. Although the average profit for those workers depends on the number of issued contracts per day, all other qualification levels have a negative profit on average regardless of the number of contracts.

Also the minimal payment per contract is a significant parameter that should be investigated in a sensitivity analysis. These results are presented in Fig. 9. Shown is the average profit for different qualification classes for a minimal payment per contract between 50 and $250 \mathrm{MU}$. Similar to the parameter investigated in Fig. 8, the average profit depends on the value of the parameter, but the main finding which qualification class performs best or worst in each variant is consistent across the whole parameter range.

Especially regarding the differences in profit for the different qualification classes, the parameter that governs the additional payment and reservation wage for each level of qualification is of crucial importance. Its influence is investigated in Fig. 10 by varying this parameter between 1 and 9 MU. Variants 2-4 are only slightly influenced by this parameter: Classes with negative income remain at negative income; classes 
with positive income are shifted to slightly higher profits. For variant 1 "Cheapest Offer", the parameter has a higher impact. If the difference in payment is very small, the profit of the different qualification classes is close together. For higher differences $(\geq$ $7 \mathrm{MU}$ ), very highly qualified workers are unable to secure any contracts. Nevertheless, the crowdworkers with the lowest qualification and thus the lowest reservation wages have the highest profit throughout the whole investigated parameter range.

This sensitivity analysis substantiates the claim that the qualitative findings of this model are not highly sensitive to specifics of the labour market or the economy. Even if we change the number of available jobs, the price of human labour or the difference in payment for differently qualified workers, the key findings about the risks and differences of the investigated variants of crowdworking remain the same. With regard to calibrating all free parameters to empirically found values, the sensitivity analysis shows that this would most likely change the quantitative results like average profits, but it is highly unlikely that the fundamental qualitative findings about the differences between the investigated crowdworking variants are influenced by such a calibration.

\section{Conclusion}

In conclusion, the presented agent-based crowdworking model was able to illustrate that there are fundamental differences between the distinct variants of crowdworking. The sensitivity analysis showed that these differences are not caused by specifics of the economy or the labour market, but rather by differences in the criteria which contract is given to which crowdworker. Thus, when investigating the risks and effects of crowdworking it is of high importance to differentiate these distinct variants, since they have a completely different behaviour regarding income distribution.

The developed model offers some unique advantages when compared to standard labour models. Similar to traditional matching models (Burgess 1994; Mortensen 2000; Eckstein and Van den Berg 2003; Yashiv 2007; Lise et al. 2016; Axtell et al. 2019), we start out from a population of workers with certain qualifications and reservation wages. However, the matching itself is fundamentally different: traditional labour models start out from equations for the unemployment rate and its derivatives and investigate stable or semi-stable states. This all happens under two assumptions that are not valid for crowdworking: (i) the assumption that once a match between job and worker is found, these two items stay matched over a longer period of time and (ii) the assumption that one job is only matched to one worker and vice versa. In crowdworking, this is not the case: all matches are short-termed and workers can be matched to several jobs simultaneously. This behaviour would be very difficult to implement in traditional models. One would have to adapt all fundamental equations of the model to account for this new type of matching. This would introduce a new problem: for the different variants of crowdworking, one would have to use different equations, since the matching is based on different principles. This would make a comparison between the variants impossible, since they would all rely on different equations and thus a different framework. By using the agent-based approach presented here, we avoid this problem. The framework is exactly the same for all variants of crowdworking; we only use a different way of matching, and even here we do not 
need to calibrate equations, because the rules of matching can be used exactly as they are in the real world. A different advantage of the presented model over traditional approaches is that we can also investigate disequilibrium dynamics. We never assume any equilibrium, we just observe a balance emerging within the agent-based model, which gives us more information than standard models would. Thus, the model was able to simulate and compare four different variants of crowdworking within the same framework, leading to the following insights.

All variants that offer a form of auction for the payment for each task, in our model variant 1 and variant 2 , tend to produce low wages. Workers are actively encouraged to bid the lowest price for which they are willing to work. Only the workers who are willing to do this have a chance to generate income in this system. This income is however generated by the sheer quantity of tasks that workers perform. The only strategy to survive in this system is to request minimal payment in order to secure as many contracts as achievable in order to work as much as possible. The overall goal of crowdworking, i.e. providing more flexibility and better working conditions compared to regular employment, is therefore very difficult to reach in this system. If the contract is not auctioned but assigned according to qualification, like in variant 4 , the situation is different. There is no way for the employee to actively cause wage dumping, since the payment is chosen by the employer. Especially workers who produce work with lesser quality have only slim chances of generating any income.

The most simple variant of crowdworking, variant 3, that uses a basic "first come, first served" system may sound unfair at first glance, but it turns out that this method has certain unique advantages. Neither employers nor workers can actively influence the prices, and the amount of contracts each worker can secure is indiscriminate of the workers qualification, reservation wage or experience. That way no individual can find a way to earn more than all other users of such a platform, leading to the situation where profit is not extremely high for some individuals and negative for others, but evenly distributed and easy to predict. Of course one should bear in mind, that this variant of crowdworking only works for very simple tasks where no minimal qualification is necessary.

When introducing global competition into the systems, the situation deteriorates more in variants that were already susceptible to wage dumping (variant 1 and variant 2). Workers with higher reservation wage and cost of living have a difficult time generating income in such a system, and also those with lower reservation wages earn more or less the bare minimum that they are willing to work for. In variants where the worker with the best qualification is chosen, national and global competitors have a similar outlook. Only some individuals can profit in such a system. Perhaps the fairest system concerning international competition is variant 3 "First Offer". Since the reservation wage has no influence on the amount of contracts one can secure and the wages for each contract are fixed, also income is distributed evenly among the crowdworkers. The only significant difference in profit is caused directly by the diverging cost of living.

Even though this analysis focuses on the situation of the crowdworkers, we can also see that the differences in crowdworking variants affect the firms and crowdworking platforms. Depending on what the firms are interested in, the different variants offer unique advantages. The variant "Cheapest Offer" leads to very low cost for the firms 
with the downside of low qualification of the workers. "Best Quality" offers access to highly qualified workers, however at a significantly higher cost. In-between those variants is the variant "Quality Requirement", leading to a platform with qualified workers at medium prices. Also the variant "First Offer" has its benefits: it is an extremely simple system, which means contracts can be fulfilled right away, without any delay, which can be an advantage for many tasks. Additionally a platform based on "First Offer" has access to a large variety of crowdworkers in terms of qualification and reservation wages, since it is not inherently biased in that regard.

An important question is of course how these findings from an abstract simulation can be translated into real economies. More specifically, what effects can we predict for the future, when crowdworking and traditional employment compete for the same workforce and shape the labour market together. Especially here it is important to differentiate between the diverse variants of crowdworking, since they show distinct behaviour.

For crowdworking that uses the variant "Cheapest Offer", we expect a significant effect on regular employment. It has a high risk of low wages, especially in a global context, (see Figs. 3, 7) possibly also affecting wages of regularly employed people, at least in areas where crowdworking is viable. Regarding the workforce, this variant seems to attract workers of low qualification (see Fig. 5), which have difficulties finding a job on the traditional labour market. They see crowdworking as a low-paying alternative that is better than unemployment and easily accessible. Thus, the impact on the available workforce is minor.

Similar effects can be expected for the variant "Quality Requirement". Because there is an incentive to work for a slightly smaller wage in order to secure more contracts, wages are small as long as there are enough active crowdworkers. In this variant, not only workers with low qualification are attracted, but also highly qualified workers have the opportunity to earn a decent living wage (see Fig. 2). Nevertheless, compared to regular employment, the incentive to underbid other workers is higher and thus average wages lower, which means that regular employment would still be preferred by most people.

In the variant "First Offer", it is not possible to underbid other crowdworkers, and thus, the wages offered by the platform are always linked to the wages offered for regular employment in the respective sector. Since workers of all qualification levels earn a similar amount (see Fig. 2) and it offers high job security (see Fig. 3) it is especially lucrative for workers with low qualification. Due to the easy access to this form of crowdworking many people could participate, leading to a relevant competition for regular employment.

In the variant "Best Quality", the aim is to attract highly qualified workers. There is no incentive for the worker to reduce their reservation wage. However, the competition with regular employment is significant. This variant specifically attracts highly qualified workers (see Fig. 5) and offers them decent wages (see Fig. 2). Especially for highly qualified workers work-life-balance, flexibility and self-determination are important factors in choosing a job and in these areas crowdworking can have obvious benefits compared to regular employment. Thus, highly qualified workers, which are already sought after on the labour market, will become even more scarce. 
In addition to this analysis that highlights the essential features of different crowdworking variants, the presented model can also be used as a platform to investigate hypothetical scenarios. It is possible to simulate a change in supply, demand or price of labour to make predictions on how the different crowdworking variants behave. Furthermore, one could expand the model to include more elaborate agent behaviour (like for example bidding below your own reservation wage to secure more contracts, invest money to increase one's qualification, or reduce one's own work quality to increase work speed) to see which strategies are successful in a crowdworking environment. It is also possible to introduce new variants of crowdworking that are currently not realized anywhere and gain insight in such systems, which would not be possible without an abstract model. In that sense, the presented model can be used complementary or supplementary to empirical studies to investigate the phenomenon crowdworking with all its associated risks and opportunities.

In order to improve the predictive power of the model, several expansions would be possible. Currently, the reservation wage of each worker is constant, as well as the compensation that the firms offer for a given contract. In principle, both these variables could be dynamic. Workers could adjust their reservation wages if they have too much or too little work. On the other hand, firms could offer more compensation, if they struggle to find suitable workers or less if there is more than enough supply. However, in the interest of being able to compare different crowdworking variants using the same foundation, we use static compensation and reservation wages. Another assumption of the model is that there is ideal information flow: the platform has access to information about the qualification of all workers all the time. Since in the real world, the information flow is more complex and often asymmetrical, it would be interesting to include such effects. Workers would need to build up reputation in order to be hired and firms with higher reputation would receive more offers. However, in this study, where we are mainly interested in the differences of the variants and less interested in individual workers or firms, these effects are neglected.

It is apparent that crowdworking can have huge advantages for workers, like more flexibility in their working time, no commuting and more self-determination, as well as benefits for the employers, like cost reduction, more flexibility concerning their workforce and a simple way to access a big human resource pool. However, there are also big risks in this context that all originate from the fact that crowdworking has no rules and regulations and can be seen as a self-regulatory, complex system. Depending on who has the biggest influence on the system (the workers, the clients, or the platform itself) the risks can be bigger or smaller. This study was able to illustrate that the most important factor in the development of a crowdworking system is how payment and the distribution of tasks are handled. Especially variants in which the crowdworkers are able to set the wages are susceptible to wage dumping. Our sensitivity analysis suggest that this phenomenon is independent of the specifics of the labour market but rather a fundamental property of those variants of crowdworking. It is important to identify the problems associated with crowdworking and their causes in order to find ways to steer these self-organizing systems on a course towards a solution that provides access to paid work for many diverse people, while limiting the possibilities for exploitation. Agent-based models provide a viable way to investigate these complex systems and 
offer the means to gain new insights for research questions where experiments or statistical analysis often have difficulties finding reliable answers.

Acknowledgements Open access funding provided by University of Graz.

Open Access This article is distributed under the terms of the Creative Commons Attribution 4.0 International License (http://creativecommons.org/licenses/by/4.0/), which permits unrestricted use, distribution, and reproduction in any medium, provided you give appropriate credit to the original author(s) and the source, provide a link to the Creative Commons license, and indicate if changes were made.

\section{References}

Amman HM, Tesfatsion L, Kendrick DA, Judd KL, Rust J (1996) Handbook of computational economics, vol 2. Elsevier, Amsterdam

Astheimer S (2015) Die Mär vom Clickworker. Frankfurter Allgemeine Zeitung

Axelrod RM (1997) The complexity of cooperation: agent-based models of competition and collaboration. Princeton University Press, Princeton

Axtell RL (2000) Why agents? on the varied motivations for agent computing in the social sciences. In: Workshop on agent simulation: applications, models, and tools. The University of Chicago, pp 1-23. https://publications.anl.gov/anlpubs/2000/09/37176.pdf\#page=12

Axtell R, Guerrero OA, López E (2019) Frictional unemployment on labor flow networks. J Econ Behav Organ 160:184-201

Balmer M, Cetin N, Nagel K, Raney B (2004) Towards truly agent-based traffic and mobility simulations. In: Proceedings of the third international joint conference on autonomous agents and multiagent systems, vol 1. IEEE Computer Society, pp 60-67

Bankes SC (2002) Agent-based modeling: a revolution? Proc Natl Acad Sciences 99(suppl 3):7199-7200

Batty M (2007) Cities and complexity: understanding cities with cellular automata, agent-based models, and fractals. The MIT Press, Cambridge

Bescherer P (2009) Prekarität, Abstieg, Ausgrenzung: Die soziale Frage am Beginn des 21. Jahrhunderts. Campus Verlag, Frankfurt

Blohm I, Leimeister JM, Krcmar H (2013) Crowdsourcing: how to benefit from (too) many great ideas. MIS Q Exec 12(4):199-211

Boeri T, Burda M, Kramarz F (2008) Working hours and job sharing in the EU and USA: are Europeans lazy? or Americans crazy? Oxford University Press, Oxford

Boero R, Squazzoni F (2005) Does empirical embeddedness matter? methodological issues on agent-based models for analytical social science. J Artif Soc Soc Simul 8(4):1-6

Bonabeau E (2002) Agent-based modeling: methods and techniques for simulating human systems. Proc Natl Acad Sci 99(suppl 3):7280-7287

Brabham DC (2008) Crowdsourcing as a model for problem solving: an introduction and cases. Convergence 14(1):75-90

Brynjolfsson E, McAfee A (2014) The second machine age: work, progress, and prosperity in a time of brilliant technologies. WW Norton \& Company, New York

Burgess SM (1994) Matching models and labour market flows. Eur Econ Rev 38(3-4):809-816

Chaturvedi A, Mehta S, Dolk D, Ayer R (2005) Agent-based simulation for computational experimentation: developing an artificial labor market. Eur J Oper Res 166(3):694-716

Chen B, Cheng HH (2010) A review of the applications of agent technology in traffic and transportation systems. IEEE Trans Intell Transp Syst 11(2):485-497

Chen X, Zhan FB (2008) Agent-based modelling and simulation of urban evacuation: relative effectiveness of simultaneous and staged evacuation strategies. J Oper Res Soc 59(1):25-33

Davidsson P (2002) Agent based social simulation: a computer science view. J Artif Soc Soc Simul 5(1):1-7

Dawid H, Gemkow S, Harting P, Neugart M (2012) Labor market integration policies and the convergence of regions: the role of skills and technology diffusion. J Evol Econ 22(3):543-562

Dawid H, Harting P, Neugart M (2014) Economic convergence: policy implications from a heterogeneous agent model. J Econ Dyn Control 44:54-80 
Deissenberg C, Van Der Hoog S, Dawid H (2008) Eurace: a massively parallel agent-based model of the European economy. Appl Math Comput 204(2):541-552

Deranty JP (2008) Work and the precarisation of existence. Eur J Soc Theory 11(4):443-463

Dessalles JL, Ferber J, Phan D (2008) Emergence in agent-based computational social science: conceptual, formal, and diagrammatic analysis. In: Yang A, Shan Y (eds) Intelligent complex adaptive systems. IGI Global, Hershey, pp 255-299

DeVaney SA (1997) Using financial ratios. In: Garman ET, Xiao JJ (eds) The mathematics of personal finance: using calculators and computers. Dame Publications, Inc., Houston, pp 141-153

Doan A, Ramakrishnan R, Halevy AY (2011) Crowdsourcing systems on the world-wide web. Commun ACM 54(4):86-96

Doniec A, Mandiau R, Piechowiak S, Espié S (2008) A behavioral multi-agent model for road traffic simulation. Eng Appl Artif Intell 21(8):1443-1454

Dosi G, Pereira MC, Roventini A, Virgillito ME (2016) The effects of labour market reforms upon unemployment and income inequalities: an agent-based model. Socio Econ Rev 16:687-720

Düll N, Bertschek I, Dworschak B, Meil P, Niebel T, Ohnemus J, Vetter T, Zaiser H (2016) Arbeitsmarkt 2030: Digitalisierung der Arbeitswelt. Fachexpertisen zur Prognose 2016. Technical report, ZEWGutachten und Forschungsberichte

Durward D, Blohm I, Leimeister JM (2016) Crowd work. Bus Inf Syst Eng 58(4):281-286

Eckstein Z, Van den Berg GJ (2003) Empirical labor search models: a survey. Technical report, working paper, IFAU-Institute for Labour Market Policy Evaluation

Emmenegger P (2012) The age of dualization: the changing face of inequality in deindustrializing societies. Oxford University Press, Oxford

Epstein JM (1999) Agent-based computational models and generative social science. Complexity 4(5):4160

Epstein JM (2006) Generative social science: studies in agent-based computational modeling. Princeton University Press, Princeton

Fagiolo G, Roventini A (2017) Macroeconomic policy in dsge and agent-based models redux: new developments and challenges ahead. J Artif Soc Soc Simul 20(1):1

Farmer JD, Foley D (2009) The economy needs agent-based modelling. Nature 460(7256):685-686

Ford M (2015) The rise of the robots: technology and the threat of mass unemployment. Oneworld Publications, London

Frey CB, Osborne MA (2017) The future of employment: how susceptible are jobs to computerisation? Technol Forecast Soc Change 114:254-280

Gilbert N (2008) Agent-based models, vol 153. Sage, London

Hofer C, Jäger G, Füllsack M (2018) Large scale simulation of $\mathrm{CO}_{2}$ emissions caused by urban car traffic: an agent-based network approach. J Clean Prod 183:1-10

Hommes CH (2006) Heterogeneous agent models in economics and finance. In: Tesfatsion L, Judd KL (eds) Handbook of computational economics, vol 2. North-Holland, Oxford, pp 1109-1186

Horton JJ, Chilton LB (2010) The labor economics of paid crowdsourcing. In: Proceedings of the 11th ACM conference on electronic commerce. ACM, pp 209-218

Howe J (2006) The rise of crowdsourcing. Wired Mag 14(6):1-4

ILO (2016) Promoting fair migration—-general survey concerning the migrant workers instruments 2016. Technical report, Committee of Experts on the Application of Conventions and Recommendations

Kaufmann N, Schulze T, Veit D (2011) More than fun and money. Worker motivation in crowdsourcing-a study on mechanical turk. AMCIS 11:1-11

Kuek SC, Paradi-Guilford C, Fayomi T, Imaizumi S, Ipeirotis P, Pina P, Singh M (2015) The global opportunity in online outsourcing. World Bank Group, Washington, DC

Leimeister JM, Zogaj S (2013) Neue Arbeitsorganisation durch Crowdsourcing: Eine Literaturstudie. Technical report, Arbeitspapier, Arbeit und Soziales

Leombruni R, Richiardi M (2006) Laborsim: an agent-based microsimulation of labour supply—an application to italy. Comput Econ 27(1):63-88

Lise J, Meghir C, Robin JM (2016) Matching, sorting and wages. Rev Econ Dyn 19:63-87

Mortensen DT (2000) Equilibrium unemployment with wage posting: Burdett-Mortensen meet pissarides. In: Bunzel H, Christensen B, Jensen P, Kiefer N, Mortensen D (eds) Panel data and structural labour market models. Emerald Group Publishing Limited, Bingley, pp 281-292

Naivinit W, Le Page C, Trébuil G, Gajaseni N (2010) Participatory agent-based modeling and simulation of rice production and labor migrations in Northeast Thailand. Environ Model Softw 25(11):1345-1358 
Neugart M, Richiardi M, et al (2012) Agent-based models of the labor market. LABORatorio R Revelli working papers series 125

OECD (2017) Harmonised unemployment rate (hur). OECD Publishing, Paris. https://doi.org/10.1787/ 52570002-en

Reheis F (2009) Entschleunigung: Abschied vom Turbokapitalismus. Riemann Verlag, München

Rest J (2015) Crowd Guru, Clickworker und Co.: Das App-Proletariat. berliner-zeitungde

Rosa H (2005) Beschleunigung: die Veränderung der Zeitstrukturen in der Moderne. Suhrkamp, Berlin

Rosa H (2010) Alienation and acceleration: towards a critical theory of late-modern temporality, vol 3. Aarhus Universitetsforlag, Aarhus

Scherschel K, Streckeisen P, Krenn M (2012) Neue Prekarität: die Folgen aktivierender Arbeitsmarktpolitikeuropäische Länder im Vergleich, vol 2. Campus Verlag, Frankfurt

Szpir M (2002) Clickworkers on mars. Am Sci 90(3):226

Tesfatsion L (2002) Agent-based computational economics: growing economies from the bottom up. Artif Life 8(1):55-82

Tesfatsion L (2006) Agent-based computational economics: a constructive approach to economic theory. In: Tesfatsion L, Judd KL (eds) Handbook of computational economics, vol 2. North-Holland, Oxford, pp 831-880

Vogler-Ludwig K, Düll N, Kriechel B, Vetter T (2015) Arbeitsmarkt 2030: Die Bedeutung der Zuwanderung für Beschäftigung und Wachstum

Yashiv E (2007) Labor search and matching in macroeconomics. Eur Econ Rev 51(8):1859-1895

Publisher's Note Springer Nature remains neutral with regard to jurisdictional claims in published maps and institutional affiliations. 